\title{
CD44 as a receptor for colonization of the pharynx by group A Streptococcus
}

\author{
Colette Cywes, ${ }^{1,2}$ Ivan Stamenkovic, ${ }^{3,4}$ and Michael R. Wessels ${ }^{1,2,5}$ \\ ${ }^{1}$ Channing Laboratory, Brigham and Women's Hospital, \\ ${ }^{2}$ Department of Medicine, and \\ ${ }^{3}$ Department of Pathology, Harvard Medical School, \\ ${ }^{4}$ Pathology Research, Massachusetts General Hospital, and \\ ${ }^{5}$ Division of Infectious Diseases, Brigham and Women's Hospital, Boston, Massachusetts, USA
}

Address correspondence to: Michael R. Wessels, Channing Laboratory, 181 Longwood Avenue,

Boston, Massachusetts 02115, USA. Phone: (617) 525-0086; Fax: (617) 731-1541; E-mail: mwessels@channing.harvard.edu.

Received for publication April 28, 2000, and accepted in revised form August 21, 2000.

The pharynx is the primary reservoir for strains of group A Streptococcus (GAS) associated both with pharyngitis (streptococcal sore throat) and with invasive or "flesh-eating" soft tissue infections. We now report that CD44, a hyaluronic acid-binding protein that mediates human cell-cell- and cellextracellular matrix-binding interactions, functions as a receptor for GAS colonization of the pharynx in vivo. We found that attachment of GAS to murine epithelial keratinocytes was mediated by binding of the GAS hyaluronic acid capsular polysaccharide to CD44. In studies of transgenic mice with a selective defect in epithelial expression of CD44, GAS adherence to CD44-deficient keratinocytes in vitro was reduced compared with adherence to keratinocytes expressing normal levels of CD44. After intranasal inoculation, GAS colonized the oropharynx of wild-type mice but failed to colonize transgenic mice deficient in CD44 expression. GAS colonization of wild-type mice could be blocked by coadministration of $\mathrm{mAb}$ to $\mathrm{CD} 44$ or by pretreatment of the animals with exogenous hyaluronic acid. These results provide evidence that CD44 serves as a receptor for GAS colonization of the pharynx and support the potential efficacy of disrupting the interaction between the GAS hyaluronic acid capsule and CD44 as a novel approach to preventing pharyngeal infection.

J. Clin. Invest. 106:995-1002 (2000).

\section{Introduction}

Streptococcus pyogenes or group A Streptococcus causes millions of infections each year throughout the world, including streptococcal pharyngitis, one of the most common bacterial infections in childhood, infections of the skin and soft tissues, and the postinfectious syndrome of acute rheumatic fever, a major cause of acquired heart disease in developing countries. In addition, epidemiological studies have documented a recent resurgence in invasive GAS infection syndromes such as necrotizing fasciitis, bacteremia, and streptococcal toxic shock syndrome (1-4). GAS colonization of the pharynx may lead not only to symptomatic sore throat, but also is an invariant antecedent to acute rheumatic fever (5). The pharynx also appears to serve as a reservoir for isolates that cause invasive infections, as GAS strains isolated from patients with invasive disease can be recovered from throat cultures of close contacts $(6,7)$.

Several GAS surface structures including lipoteichoic acid, $M$ protein, fibronectin binding proteins, and the GAS capsular polysaccharide have been implicated in adherence of the bacteria to epithelial cells in vitro (reviewed in ref. 8). However, the role of some of these potential adhesins may be limited in several ways. M protein, for example, occurs in more than 100 distinct serotypes and the degree to which $M$ protein contributes to adherence may be serotype or strain depend- ent (9-11). Fibronectin-binding proteins can mediate attachment to integrins on epithelial cells, but are expressed only on certain strains of GAS (12). Furthermore, the interaction of $\mathrm{M}$ protein and perhaps of other potential adhesins with their receptors on epithelial cells may be prevented by a masking effect of the capsular polysaccharide $(10,13)$. In contrast, the GAS capsular polysaccharide is invariant in structure and is highly conserved among GAS isolates $(14,15)$. Experimental infection studies in mice have shown that mutant strains of GAS deficient in capsular polysaccharide fail to cause infection after intranasal inoculation $(16,17)$. Because the capsular polysaccharide forms an outermost layer on the bacterial surface, it is likely to be important in adhesive interactions between the bacterial cell and the pharyngeal epithelium.

The GAS capsular polysaccharide is composed of hyaluronic acid, a high-molecular-weight polysaccharide that is structurally identical to the material found on mammalian cell surfaces and as a constituent of extracellular matrix and connective tissue (18). Binding of mammalian hyaluronic acid to the hyaluronic acidbinding protein CD44 mediates a variety of cell-cell and cell-matrix interactions including lymphopoiesis, lymphocyte homing, and tumor metastasis (19-21). The observation that the GAS capsular polysaccharide is a molecular mimic of mammalian hyaluronic acid sug- 
gested the hypothesis that colonization of the pharynx by GAS reflects subversion of the CD44-hyaluronic acid binding interaction through CD44-mediated binding of the GAS capsular polysaccharide. To test this hypothesis, we used an experimental system in mice to examine the interaction of GAS with CD44 on the pharyngeal epithelium in vitro and in vivo.

\section{Methods}

Bacterial strains and growth conditions. GAS strain B514$\mathrm{Sm}$ is a spontaneous streptomycin-resistant derivative of $\mathrm{B} 514 / 33$, an $\mathrm{M}$ type 50 strain originally isolated from an epizootic infection of a mouse colony $(22,23)$. UAB039 is an acapsular mutant of B514-Sm constructed by insertion of a nonreplicating plasmid within the has $A$ (hyaluronate synthase) gene (17). GAS strain 950771 is an M type 3 strain originally isolated from a patient with necrotizing fasciitis; strain 188 is an acapsular mutant of 950771 constructed by insertion of the $\Omega \mathrm{Km} 2$ element, a kanamycin-resistance cassette flanked by transcriptional terminators, within the has $A$ gene (24). Bacteria were grown in liquid culture in Todd-Hewitt broth or on trypticase soy agar containing $5 \%$ sheep blood. GAS was grown in liquid culture to mid-exponential phase $\left(\mathrm{A}_{650 \mathrm{~nm}}=0.15\right)$, washed, and resuspended in serum-free keratinocyte basal medium without calcium or supplements (Clonetics Inc., San Diego, California, USA) for in vitro attachment assays or in PBS ( $\mathrm{pH} 7.4$ ), for intranasal inoculation of mice.

$m A b$ 's. The mAb's used in this work were KM81, a rat anti-mouse CD44 mAb (20), or 4H1, an isotypematched control $\mathrm{mAb}$ directed to Psendomonas aeruginosa lipopolysaccharide (donated by M. Preston, Brigham and Women's Hospital). Both antibodies were purified by protein $\mathrm{G}$ affinity chromatography (GammaBind Plus Sepharose; Amersham Pharmacia Biotech Inc., Piscataway, New Jersey, USA) before use.

Mouse strains. Unless otherwise specified, experiments involving mice were performed using C57BL/6 mice (The Jackson Laboratory, Bar Harbor, Maine, USA). K5-CD44 transgenic mice with a selective deficiency in expression of CD44 in stratified squamous epithelia have been described previously (25). These mice express a CD44-antisense transgene under the control of the keratin-5 promoter that targets expression of the antisense transgene to the basal cell compartment of stratified squamous epithelia.

Derivation and characterization of primary mouse keratinocytes. Primary mouse keratinocyte cultures were established from cells isolated from the epidermis of 1to 3-day-old mice. Mouse skins were incubated overnight at $4{ }^{\circ} \mathrm{C}$ in $0.25 \%$ trypsin. The epidermis was separated from the dermis and minced and stirred to produce a single-cell suspension before seeding onto collagen-coated tissue cultures wells (Nalge Nunc International, Naperville, Illinois, USA). Cell cultures were incubated at $34^{\circ} \mathrm{C}$ in $8 \% \mathrm{CO}_{2}$ for $3-10$ days in serum-free keratinocyte basal medium without calcium, supplemented with KGM SingleQuots, original formula (Clonetics Inc.). CD44 expression on keratinocytes from each animal was assessed by immunofluorescence microscopy using mAb KM81 (10).

Bacterial adherence assays. For bacterial adherence assays, keratinocytes were seeded at $10^{5}$ cells per well in collagen-coated tissue culture wells (Nalge Nunc International), incubated for 3 days at $34^{\circ} \mathrm{C}$ in $8 \%$ $\mathrm{CO}_{2}$, washed, and overlaid with medium containing $10^{6} \mathrm{CFU}$ of GAS per well. After incubation for 1 hour at $34^{\circ} \mathrm{C}$ in $8 \% \mathrm{CO}_{2}$, the keratinocyte monolayers were washed twice to remove nonadherent bacteria, then the number of cell-associated bacteria was determined by quantitative culture after releasing the keratinocytes with trypsin and lysing them in sterile water. Experiments testing the effects of exogenous hyaluronic acid on GAS adherence were performed using the mouse keratinocyte cell line, PAM 2.12 (26). Cells were seeded in collagen-coated tissue culture wells at $10^{5}$ cells per well, cultured for 3 days at $37^{\circ} \mathrm{C}$ in $5 \% \mathrm{CO}_{2}$, and then inoculated with $10^{6} \mathrm{CFU}$ of GAS per well in medium containing exogenous hyaluronic acid (from rooster comb; Sigma Chemical Co., St. Louis, Missouri, USA), no inhibitor, or a control polysaccharide, alginic acid, a high-molecular-weight polymer of mannuronic acid and guluronic acid (Protonal Durvillea Alginate; Pronova Biopolymer Inc., Portsmouth, New Hampshire, USA). Cells were incubated for 45 minutes at $37^{\circ} \mathrm{C}$ in $5 \% \mathrm{CO}_{2}$ and then processed for enumeration of adherent bacteria as already described here. In experiments to test whether exogenous hyaluronic acid could promote dissociation of GAS attached to keratinocytes, GAS was allowed to attach to keratinocytes in the absence of inhibitor; after 45 minutes, the medium was removed and replaced with fresh medium containing hyaluronic acid, no inhibitor, or alginic acid. After 45 minutes of incubation, the number of adherent bacteria was enumerated as already described here.

Pharyngeal colonization studies in vivo. For pharyngeal colonization experiments, 4- to 6-week-old female mice were anesthetized by inhalation of methoxyflurane, then inoculated intranasally with approximately $5 \times$ $10^{6} \mathrm{CFU}$ of GAS in $20 \mu \mathrm{l} \mathrm{PBS}, \mathrm{pH}$ 7.4. Throat swabs were collected from anesthetized mice daily and were plated on Todd-Hewitt-blood agar containing streptomycin $500 \mu \mathrm{g} / \mathrm{ml}$ to inhibit growth of normal flora. For some experiments, the bacterial inoculum was suspended in PBS containing $50 \mu \mathrm{g}$ of $\mathrm{mAb}$, either KM81 directed to CD44 or an isotype-matched, irrelevant control mAb, 4H1. Examination by immunofluorescence microscopy of GAS cells incubated with $\mathrm{mAb}$ revealed no binding of either $\mathrm{mAb}$ to the organisms.

Immunobistochemistry. Paraffin-embedded tissue sections through the mouse pharynx were deparaffinized as described previously (10). Sections were incubated with $0.1 \%$ hydrogen peroxide to quench endogenous peroxidase activity, then incubated for 1 hour with $1.5 \%$ normal rabbit serum, and then with $\mathrm{mAb} \mathrm{KM} 81,15 \mu \mathrm{g} / \mathrm{ml}$, for 30 minutes at room tem- 
Figure 1

Inhibition of GAS attachment to normal mouse keratinocytes by mAb to CD44. Data represent mean \pm SD of adherent bacteria recovered after inoculation of keratinocytes with GAS in the presence of anti-CD44 mAb KM81 (hatched bars), an irrelevant control mAb $4 \mathrm{H} 1$ (open bars), or no antibody (filled bars). Treatment with $\mathrm{KM} 81$, but not with the control mAb, inhibited attachment of wild-type (encapsulated) GAS strains B514-Sm and 950771 (a). KM81 had no significant effect on binding of the acapsular mutant strain 188 (b) $\mathbf{a}$

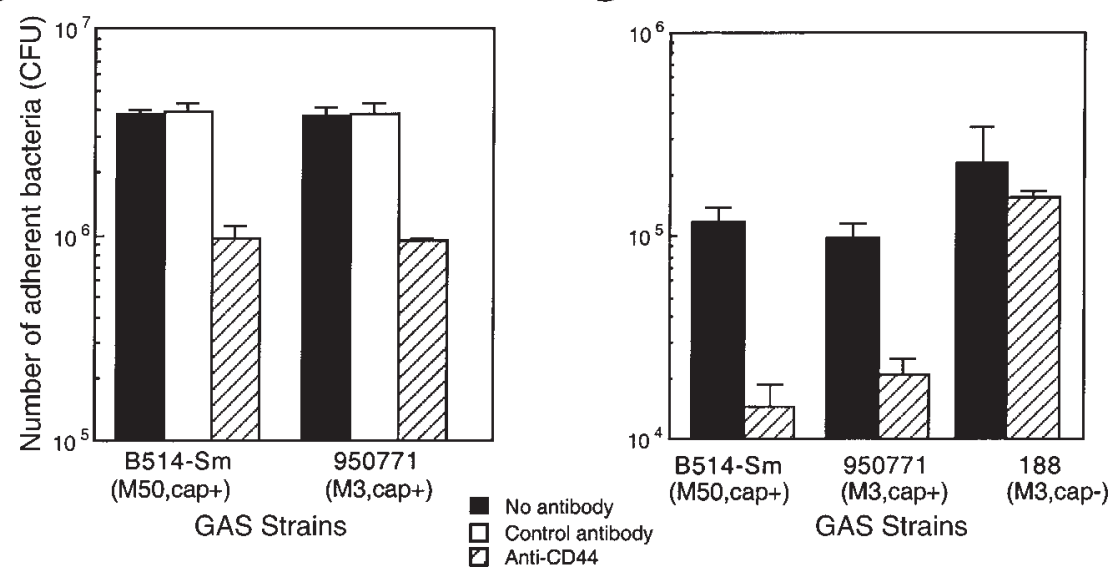

perature. Slides were washed with PBS, then incubated with biotin-conjugated rabbit anti-rat IgG, followed by horseradish peroxidase-streptavidin and the peroxidase substrate, diaminobenzidine tetrahydrochloride (Santa Cruz Biotechnology Inc., Santa Cruz, California, USA). Slides were examined by light microscopy and photographed at $400 \times$ magnification under standard conditions.

Statistical analysis. Differences in attachment of GAS to keratinocytes were evaluated using the Mann Whitney $U$ test (Instat version 1.12; GraphPad Software for Science Inc., San Diego, California, USA). For in vivo studies of pharyngeal colonization in transgenic mice, Fisher's exact test was used to evaluate the significance of differences between groups in the proportion of animals with positive throat cultures on day 4 or 5 (Instat version 1.12). For studies of blocking colonization in wild-type mice, repeated measures logistic regression was used to test for the effect of anti-CD $44 \mathrm{mAb}$ or exogenous hyaluronic acid treatment on the proportion of mice with positive throat cultures over 3 days of observation (27).

\section{Results}

GAS attachment to murine keratinocytes in vitro is mediated by binding of the GAS capsular polysaccharide to CD44. A previous study from this laboratory demonstrated that attachment of GAS to human keratinocytes in vitro was mediated by binding of the GAS hyaluronic acid capsular polysaccharide to CD44 on the keratinocyte cell membrane (10). In the present work, we sought to define further the importance of GAS interaction with CD44 in pharyngeal colonization in vivo. In contrast to the attachment of GAS to cultured keratinocytes in vitro, the binding interaction in vivo may be affected by such factors as reduced expression of CD44 in the fully differentiated epithelium, interference by the mucous layer coating the pharynx, or changes in expression of bacterial surface molecules induced by the host environment. To determine whether a murine model would be suitable for this investigation, we performed initial experiments to establish that GAS attachment to primary cultured murine keratinocytes, like that to human keratinocytes, was mediated by binding of the GAS capsular polysaccharide to CD44.

To examine the role of CD44 in GAS binding to murine keratinocytes, primary cultures were established of cells isolated from neonatal C57BL/6 mouse skin. Two GAS strains were studied: B514-Sm, an Mtype 50 strain that has been shown to colonize efficiently the upper airway of mice after intranasal inoculation, and 950771, an M-type 3 isolate originally cultured from a patient with necrotizing fasciitis and typical of strains that cause human pharyngitis and invasive infection. $\mathrm{mAb}$ KM81 directed to the

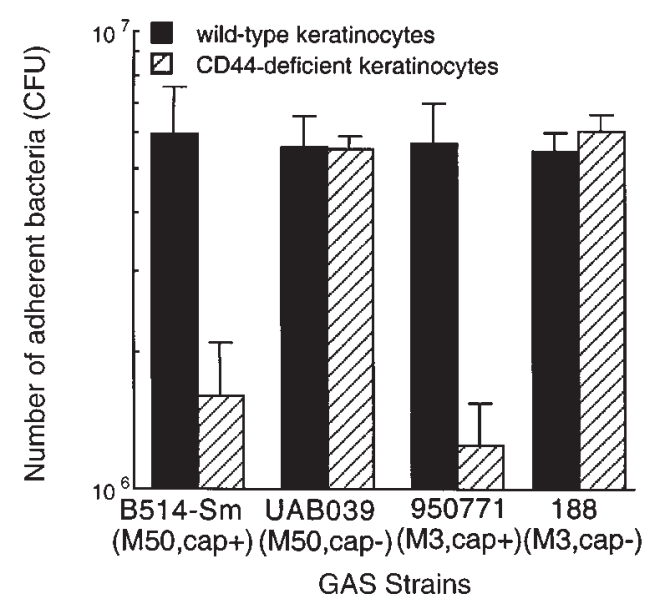

Figure 2

GAS attachment to wild-type or CD44-deficient mouse keratinocytes. Data represent mean \pm SD of adherent bacteria recovered after inoculation of wild-type (filled bars) or K5-CD44 antisense (CD44-deficient; hatched bars) keratinocytes with wild-type (encapsulated) GAS strain B514-Sm or 950771 or with their respective acapsular mutants, UAB039 and 188. Attachment of the wild-type GAS strains, but not the acapsular mutant strains, to CD44-deficient keratinocytes was reduced by approximately $75 \%$ compared with attachment to wild-type keratinocytes. 
a

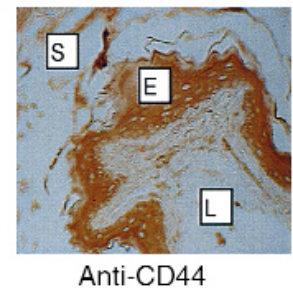

C

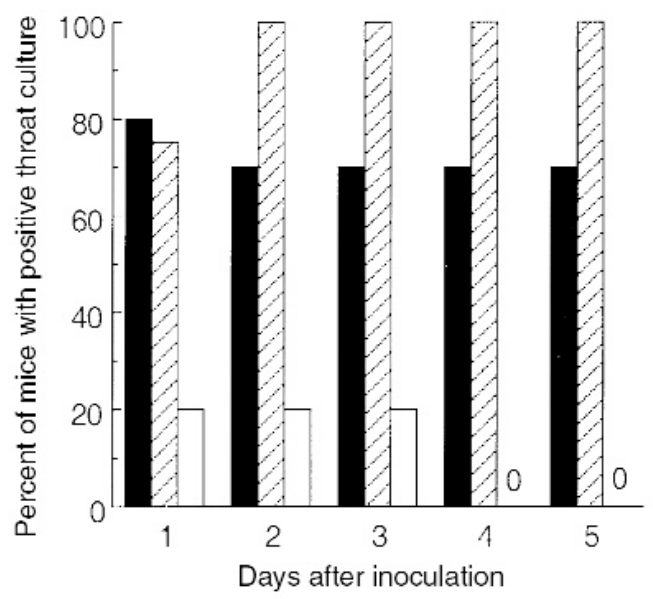

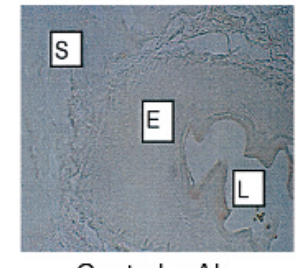

Control mAb b

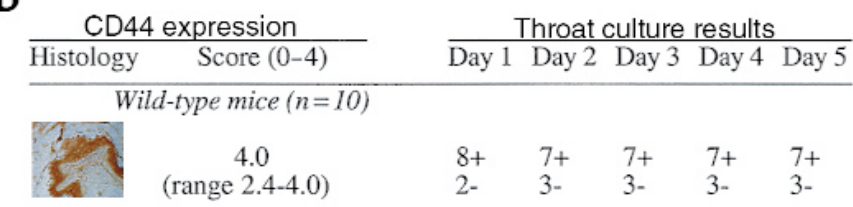

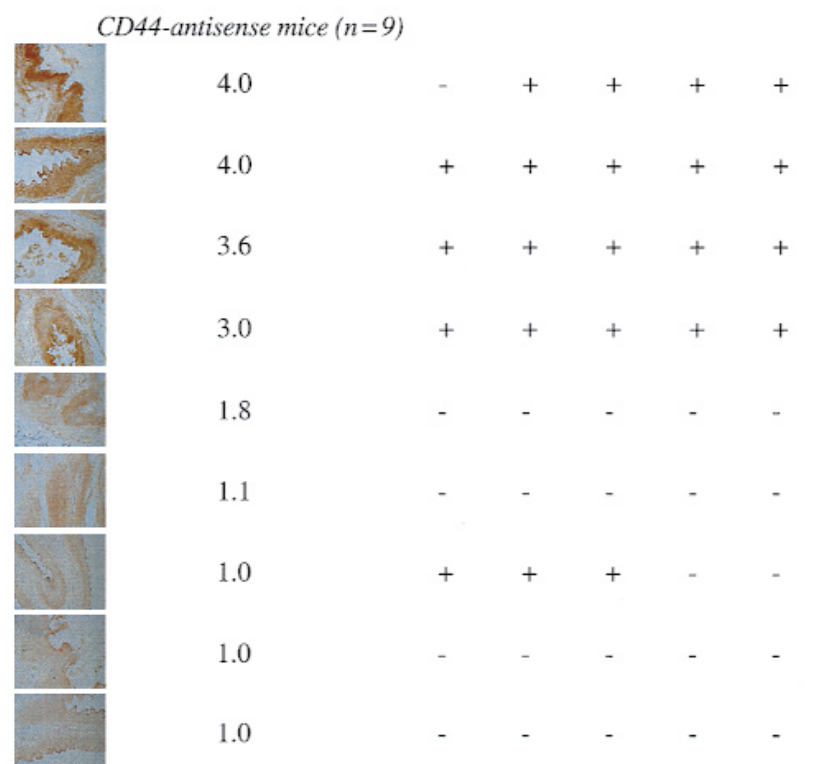

Figure 3

GAS pharyngeal colonization of wild-type mice and of mice deficient in expression of epithelial CD44. (a) Histological section through the pharynx of a representative wild-type mouse stained with mAb to CD44. Immunohistochemical staining of the pharyngeal epithelium is seen with $m A b$ to $C D 44$, but not with an irrelevant control mAb. Labels indicate the location of the epithelium (E), lumen ( $L$ ), and submucosa (S). (b) The left column shows the level of CD44 expression in the pharyngeal epithelium of wild-type and K5-CD44 antisense mice. Histological sections were scored for CD44 expression in the pharyngeal epithelium by three independent observers without knowledge of the throat culture results. CD44 expression was graded on a scale of 1 (background) to 4 (equivalent to wild-type control). On the right are results of throat cultures for GAS on each of 5 days after intranasal inoculation with GAS B514-Sm. (c) Summary of throat culture results presented in $\mathbf{b}$. Data represent the percentage of mice with a positive throat culture on each day after intranasal inoculation for wild-type mice (filled bars), transgenic mice with wild-type levels of CD44 on keratinocytes (hatched bars), and transgenic mice with reduced or absent CD44 expression on keratinocytes (open bars).

hyaluronic acid binding site of mouse CD44 (28) inhibited by more than $75 \%$ binding of wild-type (i.e., encapsulated) GAS strains B514-Sm and 950771 to mouse keratinocytes $(P<0.005$ for both GAS strains compared with binding in the presence of an irrelevant control antibody or in the absence of mAb; Figure 1). mAb KM81 had no significant effect on binding of strain 188, an isogenic acapsular mutant of strain 950771 derived from the wild-type GAS strain by inactivation of the hyaluronate synthase gene, has A. The overall adherence of the capsule-deficient strain was equal to or greater than that of the encapsulated wildtype strain, but was mediated by binding interactions independent of CD44. These results are consistent with previous studies that showed that the hyaluronic acid capsule masks alternative adhesins on the bacterial surface and prevents $M$ protein-mediated attachment of GAS to human epithelial cells $(10,13)$.

Encapsulated GAS attach poorly to CD44-deficient keratinocytes from transgenic mice. We used CD44-deficient transgenic mice to determine whether the selective loss of CD44 expression by keratinocytes affected GAS binding to keratinocytes in vitro and GAS colonization of the pharynx in vivo. K5-CD44 mice express a CD44-antisense transgene under the control of the keratin-5 promoter that targets expression of the antisense transgene to the basal cell compartment of stratified squamous epithelia (25). High-level expression of the transgene results in complete loss of CD44 expression in all layers of the epidermis. Transgenic animals were screened for high-level expression of the antisense transgene by immunofluorescence microscopy of primary cultured keratinocytes using mAb KM81. The attachment of GAS to primary keratinocytes from wild-type C57BL/6 mice was compared to GAS attachment to K5-CD44 keratinocytes from antisense transgenic animals deficient in CD44 expression. In assays of GAS attachment in vitro, binding of wild-type GAS strains B514-Sm and 950771 to CD44-deficient keratinocytes was reduced by $73 \%$ and $78 \%$, respectively, compared with binding to normal mouse keratinocytes $(P<0.01$ for binding 
of both GAS strains to wild-type versus CD44-deficient keratinocytes; Figure 2). In contrast, binding of each of two isogenic GAS mutant strains lacking hyaluronic acid capsules to CD44-deficient keratinocytes was no different than that to normal keratinocytes. These results provide further evidence that attachment of wild-type (i.e., encapsulated) GAS to mouse keratinocytes in vitro requires CD44.

GAS colonization of the airway is impaired in CD44-deficient transgenic mice in vivo. In vivo challenge experiments were performed to determine whether the observed in vitro interaction between the GAS capsule and CD44 reflected a role for CD44 as a receptor for GAS colonization of the pharyngeal epithelium in vivo. Groups of wild-type or K5-CD44 transgenic mice were inoculated intranasally with GAS strain B514$\mathrm{Sm}$. Throat cultures were obtained daily for 5 days after challenge to assess pharyngeal colonization. The degree of suppression of CD44 expression may be partial or complete in an individual animal depending on the extent of expression of the K5-CD44 antisense transgene. Therefore, the mice were euthanized at the end of the experiment, and histological sections of the pharynx were examined by microscopy after immunohistochemical staining to determine the degree of CD44 expression in the pharyngeal epithelium in individual animals. Four of nine evaluable transgenic mice exhibited epithelial expression of CD44 similar to the levels in wild-type mice (histological scores for CD44 expression of 3.0-4.0 vs. 2.4-4.0 for control mice). In five transgenic animals, epithelial CD44 expression was markedly reduced or undetectable (histological scores of 1.0-1.8). Among the wild-type mice, throat cultures were positive for GAS in eight of ten animals 1 day after inoculation and in seven of ten animals on days 2-5 (Figure 3). Similar results were observed in the transgenic animals with wild-type levels of CD44 expression: throat cultures were positive in three or four of four mice on each of 5 days after challenge. By contrast, among the five transgenic mice with low or absent epithelial CD44 expression, only a single animal had a positive throat culture on days 1, 2, and 3, and all five animals had negative cultures on days 4 and 5 (Figure 3; $P<0.03$ for comparison to CD44-positive transgenic mice or to wild-type mice). Therefore, reduced CD44 expression on the pharyngeal epithelium was associated with rapid clearance of the GAS inoculum from the upper airway and failure of the bacteria to colonize the pharynx.

Blocking GAS binding to CD44 prevents GAS pharyngeal colonization in wild-type mice. The experiments with transgenic mice described here indicated that pharyngeal expression of CD44 resulted in enhanced colonization by GAS. As an alternative means to characterize the role of CD44 as a GAS receptor, we investigated the effect of disrupting the interaction between the GAS capsule and CD44 in wild-type mice. For these studies, wild-type mice were inoculated intranasally with GAS strain B514-Sm mixed with either mAb to
CD44 or an irrelevant control mAb directed to the lipopolysaccharide of $P$. aeruginosa. Intranasal administration of anti-CD44 mAb KM81, together with the GAS challenge, reduced colonization by approximately $60 \%$ on each of 3 days after inoculation compared with coadministration of the control antibody with the bacterial inoculum $(P<0.0001$; Figure 4$)$. Thus, antibody specific for the hyaluronate-binding domain of the CD44 receptor blocked not only GAS attachment to epithelial cells in vitro, but also pharyngeal colonization by GAS in vivo.

Exogenous byaluronic acid blocks GAS binding to murine keratinocytes. To investigate the specificity of the interaction between the GAS capsule and CD44 on epithelial cells, we tested whether exogenous hyaluronic acid could compete with GAS for binding to murine keratinocytes. Addition of exogenous hyaluronic acid inhibited GAS attachment to the murine keratinocyte cell line PAM2.12 by more than 95\% $(P<0.03$; Figure 5). Another high-molecular-weight acidic polysaccharide was used as a control for nonspecific effects through which hyaluronic acid might inhibit GAS attachment, independent of interaction with CD44. Such effects might include modification of bacterial-host cell interactions mediated by electrostatic forces, or interference with bacterial attachment by viscosity of the polysaccharide solution. Accordingly, we used as a control alginic acid, another uronic acid-containing, high-molecular-weight polysaccharide with similar viscosity to hyaluronic acid. Alginic acid had no effect, supporting the hypothesis that adherence depends on a specific interaction between the GAS hyaluronic acid capsule and CD44. Addition of exogenous hyaluronic acid up to 45 minutes after adding GAS to the keratinocytes still resulted in a greater than $90 \%$ reduction in bacterial attachment $(P<0.03)$; if addition of hyaluronic acid was delayed for 2 hours, GAS attachment was
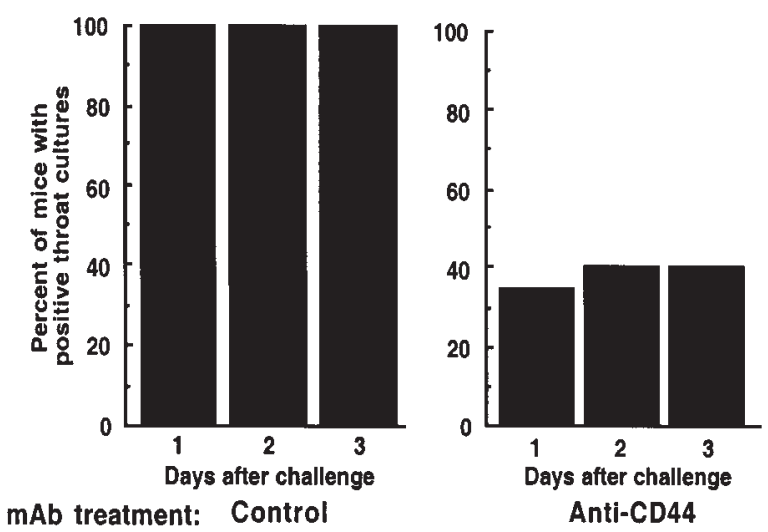

Figure 4

Prevention of GAS pharyngeal colonization in vivo by anti-CD44 $m A b$. Data represent the fraction of mice with positive throat cultures for GAS on each of 3 days after intranasal inoculation with GAS administered either with $\mathrm{mAb}$ to CD44 or with an irrelevant control $\mathrm{mAb}(n=20$ mice per group). 
Figure 5

Inhibition of GAS attachment to mouse keratinocytes by exogenous hyaluronic acid. Data represent mean \pm SD of adherent bacteria recovered after incubation of keratinocytes with GAS in the presence of no inhibitor, hyaluronic acid (HA), or alginic acid $(A L)$ at the indicated times after addition of GAS to the keratinocytes.
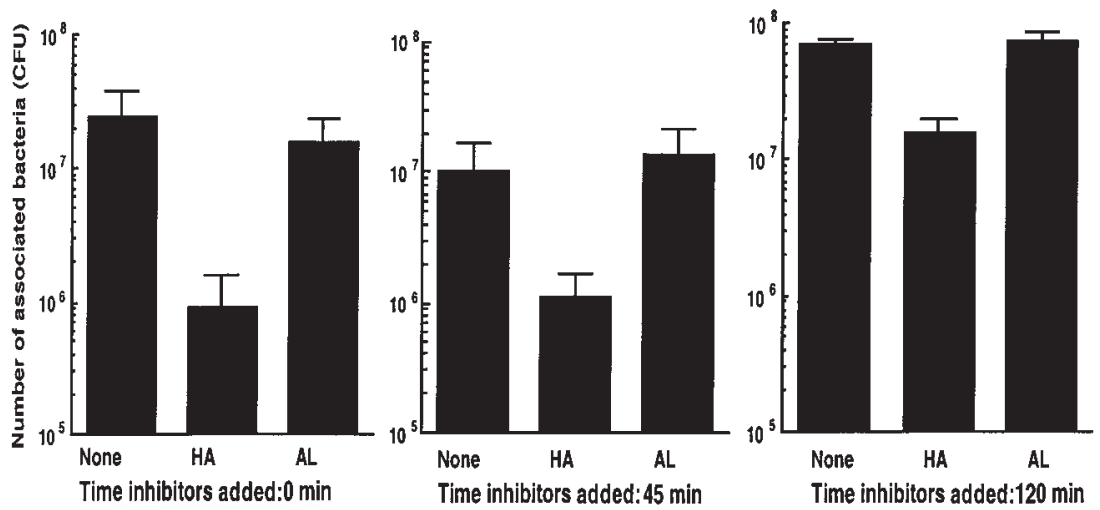

reduced by $70-80 \%(P<0.03)$. These results suggest that the capsule-CD44 binding interaction may be most important during the early phase of bacterial attachment, whereas alternative ligand-receptor interactions supervene during the later phase.

Pretreatment of mice with exogenous byaluronic acid reduces GAS colonization of the pharynx. The experiments described here indicated that exogenous hyaluronic acid could compete for GAS binding to epithelial cells in vitro. We tested whether the same phenomenon might operate in vivo in the mouse model of pharyngeal colonization. Mice were pretreated by intranasal administration of hyaluronic acid $(20 \mu \mathrm{l}$ of a $1 \mathrm{mg} / \mathrm{ml}$ solution) 5 hours before intranasal inoculation with GAS. Colonization of hyaluronic acid pretreated animals was reduced by approximately $60-80 \%$ compared with control animals pretreated with PBS or animals pretreated with alginic acid $(P<0.0001$; Figure 6$)$. That hyaluronic acid pretreatment can reduce colonization upon bacterial challenge further supports the hypothesis that interaction with CD44 mediates GAS attachment to the pharyngeal mucosa. Furthermore, it suggests the possibility that topically administered hyaluronic acid or an analog that binds to CD44 might be an effective means of preventing GAS pharyngeal infection during a period of intensive exposure such as an outbreak or epidemic situation.

\section{Discussion}

Although a great many ligand-receptor interactions have been suggested to play a role in pathogenesis of infection by various microorganisms, there are relatively few for which rigorous experimental evidence supports a significant role in vivo. The

\section{Figure 6}

Prevention of GAS pharyngeal colonization by pretreatment with hyaluronic acid. Data represent the fraction of mice with positive throat cultures for GAS on each of 3 days after intranasal inoculation with GAS following pretreatment with PBS, hyaluronic acid $(\mathrm{HA})$, or alginic acid (AL) ( $n=13$ mice per group). present investigation provides evidence that the hyaluronate-binding protein CD44 acts as a receptor for attachment of GAS to pharyngeal epithelial cells in vitro and in vivo, results consistent with in vitro studies of GAS attachment to human keratinocytes (10). Several GAS surface molecules in addition to the hyaluronic acid capsule have been implicated in adherence of the organisms to epithelial cells; however, these alternative adhesins failed to mediate effective colonization when the interaction between the hyaluronic acid capsule and epithelial CD44 was perturbed by a blocking antibody, by exogenous hyaluronic acid, or by reduced epithelial expression of CD44. Strains of GAS that lack a hyaluronic acid capsule adhere to epithelial cells in vitro at least as well as encapsulated strains; however, capsule-deficient strains colonize the pharynx poorly in vivo and are avirulent in experimental infection models $(16,17,29)$. Therefore, effective colonization of the pharynx by virulent strains of GAS appears to require the specific interaction of the GAS capsular polysaccharide with CD44.

One limitation of the present study is that results obtained in mice are not necessarily an accurate reflection of the interaction of GAS with the human host. GAS is primarily a human pathogen; many strains associated with human pharyngitis are relatively inefficient at infecting the airway of mice. Conversely, the mouse-virulent strain B514-Sm used in our in vivo studies is not entirely representative of most clinical isolates of GAS in that expression of the
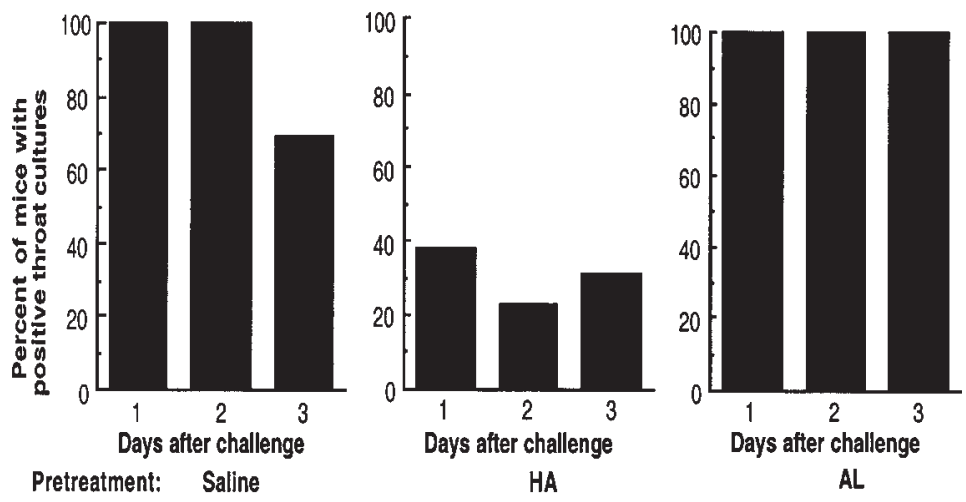
mga-regulated genes including $\mathrm{M}$ protein is very low (30). However, previous studies of diverse clinical isolates of GAS have shown that interaction between the hyaluronic acid capsule and CD44 plays an important role in attachment of these strains to human pharyngeal epithelial cells in vitro (10).

The GAS capsular polysaccharide is only one of several surface molecules that participate in GAS attachment to human cells. The presence of the capsule does not increase overall adherence, and, in some cases, encapsulated strains exhibit decreased adherence compared to isogenic acapsular mutants. Clinical isolates of GAS vary widely with respect to the type and quantity of surface molecules they express. Thus, for highly encapsulated strains, the interaction between the capsular polysaccharide and CD44 may be critical for attachment, whereas for poorly encapsulated strains, alternative bacterial ligands such as $\mathrm{M}$ protein, fibronectin-binding proteins, lipoteichoic acid, glyceraldehyde-3-phosphate dehydrogenase, and/or surface enolase may play a more important role. However, studies in a primate model demonstrated that although a capsule-deficient strain of GAS colonized the pharynx with similar efficiency to an isogenic encapsulated strain, the acapsular strain was subsequently cleared, although animals challenged with the encapsulated wild-type strain remained persistently colonized (29). Thus, although poorly encapsulated strains may adhere to the pharyngeal epithelium via CD44-independent mechanisms, such strains are susceptible to early clearance by the host, perhaps because of their enhanced susceptibility to complement-mediated phagocytic killing $(29,31,32)$.

Hasty et al. have suggested that GAS adherence to host cells may involve an initial phase of attachment mediated by relatively weak binding between bacterial ligands and their receptors on the host cell, followed by a later phase of more avid binding mediated by additional ligand-receptor interactions (33). Our finding that exogenous hyaluronic acid interferes with GAS attachment to keratinocytes most effectively during the first 45 minutes of bacterial association with the host cells is compatible with this two-step model and suggests that the GAS capsule functions as an adhesin during the early phase of bacterial adherence.

CD44-mediated attachment may be important not only in the initial interaction of the bacteria with the pharyngeal mucosa, but also in persistence and invasion into deeper tissues, as CD44 expression on keratinocytes is increased after cell injury and during wound healing $(34,35)$. Thus, binding of the GAS hyaluronic acid capsule to CD44 on epithelial cells is likely to be enhanced by local tissue injury from GAS secreted products such as cysteine protease and streptolysin $\mathrm{O}$ or as a result of the host inflammatory response to infection. In addition, the interaction of hyaluronic acid or its fragments with CD44 has been shown to regulate expression of transcription factors in endothelial cells, to induce expression of inflammatory chemokines in macrophages, and to induce nitric oxide synthase expression in macrophages and Kuppfer cells (36-39). Similarly, binding of the GAS capsular polysaccharide to CD44 on pharyngeal keratinocytes or other cell types may evoke or modulate specific cellular responses such as inflammatory cytokine expression or induction of apoptosis, responses that influence the evolution of systemic GAS disease syndromes such as streptococcal toxic shock syndrome or postinfectious autoimmune sequelae including acute rheumatic fever.

The recognition of CD44 as a receptor for a major microbial pathogen adds a new dimension to the multifaceted role of CD44 in cell-cell communication. The interaction between the GAS capsular polysaccharide and CD44 is a striking example of microbial adaptation to survival within the host through subversion of a host intercellular communication pathway. Interventions designed to disrupt that interaction represent a novel potential approach to prevention of GAS infection.

\section{Acknowledgments}

We thank H. Warren and E. Meluleni for assistance with preparation and analysis of the histology samples, S. Andreadis for assistance with breeding and genotypic screening of transgenic mice, M. Preston for providing $\mathrm{mAb} 4 \mathrm{H} 1, \mathrm{~V}$. Carey for assistance with statistical analysis of the data, and G. Pier and D. Kasper for critical review of the manuscript. This work was supported by NIH grant AI29952, NIH contract AI75326, and by a grant-in-aid from the American Heart Association.

1. Stevens, D.L., et al. 1989. Severe group A streptococcal infections associated with a toxic shock-like syndrome and scarlet fever toxin A. $N$. Engl. J. Med. 321:1-8.

2. Hoge, C.W., et al. 1993. The changing epidemiology of invasive group A streptococcal infections and the emergence of streptococcal toxic shock-like syndrome. JAMA. 269:384-389.

3. Carapetis, J., Robins-Browne, R., Martin, D., Shelby-James, T., and Hogg, G. 1995. Increasing severity of invasive group A streptococcal disease in Australia: clinical and molecular epidemiological features and identification of a new virulent M-nontypeable clone. Clin. Infect. Dis. 21:1220-1227.

4. Kaul, R., et al. 1997. Population-based surveillance for group A streptococcal necrotizing fasciitis: clinical features, prognostic indicators, and microbiologic analysis of seventy-seven cases. Am. J. Med. 103:18-24.

5. Massell, B.F. 1997. Rheumatic fever and streptococcal infection. Harvard University Press. Boston, Massachusetts, USA. 394 pp.

6. Davies, H.D., et al. 1996. Invasive group A streptococcal infections in Ontario, Canada. Ontario Group A Streptococcal Study Group. N. Engl. J. Med. 335:547-554.

7. Cockerill, F.R., III, et al. 1997. An outbreak of invasive group A streptococcal disease associated with high carriage rates of the invasive clone among school-aged children. JAMA. 277:38-43.

8. Jenkinson, H.F., and Lamont, R.J. 1997. Streptococcal adhesion and colonization. Crit. Rev. Oral Biol. Med. 8:175-200.

9. Courtney, H.S., Liu, S., Dale, J.B., and Hasty, D.L. 1997. Conversion of M serotype 24 of Streptococcus pyogenes to M serotypes 5 and 18 : effect on resistance to phagocytosis and adhesion to host cells. Infect. Immun. 65:2472-2474.

10. Schrager, H.M., Albertí, S., Cywes, C., Dougherty, G.J., and Wessels, M.R. 1998. Hyaluronic acid capsule modulates M protein-mediated adherence and acts as a ligand for attachment of group A Streptococcus to CD44 on human keratinocytes. J. Clin. Invest. 101:1708-1716.

11. Berkower, C., Ravins, M., Moses, A.E., and Hanski, E. 1999. Expression of different group A streptococcal $\mathrm{M}$ proteins in an isogenic background demonstrates diversity in adherence to and invasion of eukaryotic cells. Mol. Microbiol. 31:1463-1475.

12. Natanson, S., et al. 1995. Distribution of fibronectin-binding proteins 
among group A streptococci of different $\mathrm{M}$ types. J. Infect. Dis. 171:871-878.

13. Courtney, H.S., Ofek, I., and Hasty, D.L. 1997. M protein mediated adhesion of $\mathrm{M}$ type 24 Streptococcus pyogenes stimulates release of interleukin-6 by HEp-2 tissue culture cells. FEMS Microbiol. Lett. 151:65-70.

14. Wessels, M.R., Goldberg, J.B., Moses, A.E., and DiCesare, T.J. 1994 Effects on virulence of mutations in a locus essential for hyaluronic acid capsule expression in group A streptococci. Infect. Immun. 62:433-441.

15. Crater, D.L., and van de Rijn, I. 1995. Hyaluronic acid synthesis operon (has) expression in group A streptococci. J. Biol. Chem. 270:18452-18458.

16. Wessels, M.R., and Bronze, M.S. 1994. Critical role of the group A streptococcal capsule in pharyngeal colonization and infection in mice. Proc. Natl. Acad. Sci. USA. 91:12238-12242.

17. Husmann, L.K., Yung, D.-L., Hollingshead, S.K., and Scott, J.R. 1997. Role of putative virulence factors of Streptococcus pyogenes in mouse models of long-term throat colonization and pneumonia. Infect. Immun. 65:1422-1430.

18. Sandson, J., Hamerman, D., Janic, R., and Rojkind, M. 1968. Immunologic and chemical similarities between the streptococcus and human connective tissue. Trans. Assoc. Am. Physicians. 81:249-257.

19. Miyake, K., Underhill, C.B., Lesley, J., and Kincade, P.W. 1990. Hyaluronate can function as a cell adhesion molecule and CD44 participates in hyaluronate recognition. J. Exp. Med. 172:69-72.

20. Miyake, K., et al. 1990. Monoclonal antibodies to Pgp-1/CD44 block lympho-hemopoiesis in long-term bone marrow cultures. J. Exp. Med. 171:477-488.

21. Yu, Q., Toole, B.P., and Stamenkovic, I. 1997. Induction of apoptosis of metastatic mammary carcinoma cells in vivo by disruption of tumor cell surface CD44 function. J. Exp. Med. 186:1985-1996.

22. Husmann, L.K., Dillehay, D.L., Jennings, V.M., and Scott, J.R. 1996. Streptococcus pyogenes infection in mice. Microb. Pathog. 20:213-224.

23. Hook, E.W., Wagner, R.R., and Lancefield, R.C. 1960. An epizootic in Swiss mice caused by a group A streptococcus, newly designated type 50. Am. J. Trop. Med. Hyg. 72:111-119.

24. Ashbaugh, C.D., Warren, H.B., Carey, V.J., and Wessels, M.R. 1998 Molecular analysis of the role of the group A streptococcal cysteine protease, hyaluronic acid capsule, and $\mathrm{M}$ protein in a murine model of human invasive soft-tissue infection. J. Clin. Invest. 102:550-560.

25. Kaya, G., Rodriguez, I., Jorcano, J.L., Vassalli, P., and Stamenkovic, I 1997. Selective suppression of CD44 in keratinocytes of mice bearing an antisense CD44 transgene driven by a tissue-specific promoter disrupts hyaluronate metabolism in the skin and impairs keratinocyte proliferation. Genes Dev. 11:996-1007.

26. Song, I.S., et al. 2000. Substance P induction of murine keratinocyte PAM 212 interleukin 1 production is mediated by the neurokinin 2 receptor (NK-2R). Exp. Dermatol. 9:42-52.

27. Zeger, S.L., and Liang, K.-Y. 1986. Longitudinal data analysis for discrete and continuous outcomes. Biometrics. 42:121-130.

28. Zheng, Z., et al. 1995. Monoclonal antibodies to CD44 and their influence on hyaluronan recognition. J. Cell Biol. 130:485-495.

29. Ashbaugh, C.D., et al. 2000. Bacterial determinants of persistent throat colonization and the associated immune response in a primate model of human group A streptococcal pharyngeal infection. Cellular Microbiology. 2:283-292.

30. Yung, D.L., and Hollingshead, S.K. 1996. DNA sequencing and gene expression of the emm gene cluster in an M50 group A streptococcus strain virulent for mice. Infect. Immun. 64:2193-2200.

31. Dale, J.B., Washburn, R.G., Marques, M.B., and Wessels, M.R. 1996. Hyaluronate capsule and surface $M$ protein in resistance to opsonization of group A streptococci. Infect. Immun. 64:1495-1501.

32. Moses, A.E., et al. 1997. Relative contributions of hyaluronic acid capsule and $\mathrm{M}$ protein to virulence in a mucoid strain of group A Streptococcus. Infect. Immun. 65:64-71.

33. Hasty, D.L., Ofek, I., Courtney, H.S., and Doyle, R.J. 1992. Multiple adhesins of streptococci. Infect. Immun. 60:2147-2152.

34. Penneys, N.S. 1993. CD44 expression in normal and inflamed skin. J. Cutan. Pathol. 20:250-253.

35. Oksala, O., et al. 1995. Expression of proteoglycans and hyaluronan during wound healing. J. Histochem. Cytochem. 43:125-135.

36. Deed, R., et al. 1997. Early-response gene signalling is induced by angiogenic oligosaccharides of hyaluronan in endothelial cells. Inhibition by non-angiogenic, high-molecular-weight hyaluronan. Int. J. Cancer. 71:251-256.

37. McKee, C.M., et al. 1996. Hyaluronan (HA) fragments induce chemokine gene expression in alveolar macrophages. The role of HA size and CD44. J. Clin. Invest. 98:2403-2413.

38. McKee, C.M., et al. 1997. Hyaluronan fragments induce nitric-oxide synthase in murine macrophages through a nuclear factor kappaBdependent mechanism. J. Biol. Chem. 272:8013-8018.

39. Rockey, D.C., Chung, J.J., McKee, C.M., and Noble, P.W. 1998. Stimulation of inducible nitric oxide synthase in rat liver by hyaluronan fragments. Hepatology. 27:86-92. 